Research Article

\title{
Evaluation of Identification and Susceptibility for Candida Spp. Isolated Directly from Positive Blood Culture Bottles
}

\author{
Ândrea Celestino de Souza $\mathbb{D}^{1},{ }^{1}$ Luciano Z. Goldani $\mathbb{D}^{2},{ }^{2}$ Eliane Würdig Roesch ${ }^{\mathbb{D}}{ }^{1}$ \\ Larissa Lutz $\mathbb{D}^{1},{ }^{1}$ Patricia Orlandi Barth $\mathbb{D}^{1},{ }^{1}$ Paulo André de Souza Sampaio ${ }^{(D)}{ }^{1}$ \\ Valério Rodrigues Aquino, ${ }^{1}$ and Dariane Castro Pereira $\mathbb{D}^{1}$ \\ ${ }^{1}$ Microbiology Unit, Hospital de Clínicas de Porto Alegre, Universidade Federal Do Rio Grande do Sul, Porto Alegre, Brazil \\ ${ }^{2}$ Infectious Diseases Section, Universidade Federal Do Rio Grande do Sul, Porto Alegre, Brazil \\ Correspondence should be addressed to Luciano Z. Goldani; lgoldani@ufrgs.br
}

Received 10 May 2021; Revised 30 August 2021; Accepted 1 October 2021; Published 16 November 2021

Academic Editor: Faham Khamesipour

Copyright (c) 2021 Ândrea Celestino de Souza et al. This is an open access article distributed under the Creative Commons Attribution License, which permits unrestricted use, distribution, and reproduction in any medium, provided the original work is properly cited.

\begin{abstract}
Determination of the susceptibility profile of isolates of Candida from blood culture bottles is extremely important for correctly guiding patient pharmacotherapy. The aim of this study was to compare the results of analysis of Candida isolated directly from blood culture bottles by the VITEK MS MALDI-TOF identification system and the fluconazole disk diffusion assay with those of standard identification methods. Testing directly from the bottle allowed results 24 to 48 hours quicker than the standard method. There was a categorical agreement of 51.64\% (47 of 91 samples) between the results of analysis directly from the bottle and analysis by the standard method. Regarding species identification, there was $96.15 \%$ agreement for Candida parapsilosis (25 of 26 samples). Categorical agreement between the rapid and standard disk diffusion methods was 95\%, and the agreement between the rapid disk diffusion method and the broth microdilution method was $97 \%$. Only minor errors in the rapid method were observed: 3 (5\%) in the standard disk diffusion method and $2(3 \%)$ in the broth microdilution method. Our study concluded that the rapid disk diffusion method for fluconazole is a fast, easy, reproducible, and consistent method. Its timely implementation for testing antifungal agents in the clinical microbiology laboratory can help reduce profile release times, thus helping to determine the most appropriate antifungal treatment.
\end{abstract}

\section{Introduction}

Bloodstream infections caused by Candida species are becoming increasingly common in hospitals, with the incidence being fivefold higher than that in the last decade, and are associated with high morbidity and mortality rates $[1,2]$. Fluconazole is a well-tolerated triazole antifungal with high bioavailability and tissue penetration ability [3]. However, prolonged treatment could induce resistant mutations in Candida that lead to therapeutic failure, which is a critical concern since fluconazole is the most commonly used antifungal agent for the prophylaxis and treatment of Candida infections in many parts of the world [1].
In a 9-year retrospective cohort study at a 1250-bed US Hospital, Kollef et al. found that the hospital mortality rate for septic shock patients who received antifungal therapy within 24 hours of candidemia onset was 52.8\% $(n=142)$, compared to $97.6 \%(n=82)$ in those who did not receive antifungal therapy [4]. Other studies have found that the 30day survival rate of candidemia patients who receive appropriate pharmacotherapy was better than that of patients who received delayed or no treatment. These studies show that late initiation of adequate pharmacotherapy in infected patients correlates with an increased mortality rate [5-7]. Thus, determining the species responsible for the infection and the susceptibility profile of Candida spp. is important not only for guiding pharmacotherapy but also for 
monitoring the treatment efficacy and the emergence of resistance.

This study aimed to use a rapid method to identify species of Candida and assess fluconazole susceptibility directly from positive blood cultures.

\section{Methods}

The study was approved by the local ethics committee.

2.1. Yeast Strains. Isolates of Candida were obtained from blood cultures of patients admitted to a tertiary care hospital in Southern Brazil. For the rapid identification method, we included all the samples of blood cultures from patients with Candida species isolated between September 2018 and June 2019. For the rapid disk diffusion method, we included only one sample per patient between September 2018 and September 2019.

Blood samples were inoculated in aerobic bottles and incubated in the BacT/ALERT ${ }^{\circledR} 3 \mathrm{D}$ system (bioMérieux, France) for microorganism growth monitoring. We excluded from the study samples from which more than one microbial species was isolated. Candida albicans ATCC 90028, Candida tropicalis ATCC 750, Candida krusei ATCC 6258, and Candida parapsilosis ATCC 90018 were included as quality control strains.

2.2. Standard Method. Following microorganism growth identification by the BacT/ALERT ${ }^{\otimes} 3 \mathrm{D}$ system, blood culture media were collected from each bottle and subjected to Gram staining. Then, samples were subcultured on solid growth media, including blood agar (bioMérieux) and Sabouraud agar (Merck, Germany), and incubated at $35^{\circ} \mathrm{C}$ for 18-24 hours. To estimate the cell numbers in the bottles, 5 positive blood culture bottles were randomly selected. Then, $1 \mathrm{~mL}$ sample was aspirated from each of these bottles, serially 10 -fold diluted with sterile saline, and $50 \mu \mathrm{L}$ of suspensions was plotted on the Sabouraud agar plate, and colonies were counted after $24 \mathrm{~h}$ of incubation (ranged from $7 \times 10^{5}$ to $5 \times 10^{7} \mathrm{CFU} / \mathrm{mL}$ ). The rapid disc diffusion method was performed according to the RAST methodology standardized by the European Committee on Antimicrobial Susceptibility Testing. Following incubation, isolated colonies were subjected to analysis by the MALDI-TOF VITEK $\mathrm{MS}^{\circledast} 3.0$ system (bioMérieux, France) according to the manufacturer's instructions. Fluconazole susceptibility was assessed using a disk diffusion method according to the CLSI M44-A2 guidelines and a broth microdilution method according to the European Committee on Antimicrobial Susceptibility Testing guidelines $[8,9]$.

2.3. Rapid Identification Method. The rapid identification method was performed according to the protocol proposed by Spanu et al. [10]. Each test was conducted in duplicate. An $8 \mathrm{ml}$ aliquot from the blood culture bottle was centrifuged at $10,000 \mathrm{rpm}$ for 2 minutes at room temperature. The supernatant was discarded, and the pellet was washed twice with $1 \mathrm{ml}$ of pure water and recentrifuged. It was suspended in $1 \mathrm{ml}$ of $0.1 \%$ Tween 80 , incubated for 2 minutes, recentrifuged, washed twice with $1 \mathrm{ml}$ of pure water, recentrifuged, suspended in $300 \mu \mathrm{l}$ of pure water plus $900 \mu \mathrm{l}$ of absolute ethanol, and recentrifuged. Then, $30 \mu \mathrm{l}$ of $70 \%$ formic acid plus $30 \mu \mathrm{l}$ of pure acetonitrile was added to the pellet, and it was thoroughly vortexed and centrifuged at $14,000 \mathrm{rpm}$ for 2 minutes. A $1 \mu \mathrm{l}$ aliquot of the supernatant was collected and applied to a steel MALDI target plate. Finally, the sample was subjected to analysis by the MALDITOF VITEK MS ${ }^{\circledR} 3.0$ system (bioMérieux, France).

2.4. Rapid Disk Diffusion Method. The rapid disk diffusion method was performed according to Jabeen et al. [11]. A $100 \mu \mathrm{L}$ aliquot from the blood culture bottles was used to make lawns on Mueller-Hinton agar supplemented with 2\% dextrose and $0.5 \mu \mathrm{g} / \mathrm{ml}$ methylene blue dye. Two discs with $25 \mu \mathrm{g}$ of fluconazole were placed on the plates, and the plates were incubated.

2.5. Statistical Analysis. The kappa coefficients and categorical agreement of the data were determined using software PASW v.18 (IBM, USA). The acceptable rate agreement was $\leq 90 \%$ (10). Errors were classified into very major errors, major errors, and minor errors, and the acceptable rates were $\leq 1.5 \%, \leq 3 \%$, and $\leq 10 \%$, respectively [12].

\section{Results}

A total of 91 blood culture samples from 46 patients were tested by rapid identification method tests. The overall agreement of Candida species identification between the rapid and standard methods was $51.64 \%$. Candida parapsilosis had the highest agreement (96.15\%) of the tested samples (Table 1). No sample containing Candida orthopsilosis or Candida pelliculosa was identified, and the agreement for other species varied from 30 to $67 \%$ (Table 1).

A total of 62 samples were used to assess fluconazole susceptibility by the standard disk diffusion method, the rapid disk diffusion method, and the broth microdilution method. Table 2 presents the susceptibility profiles of the Candida species isolated in the study obtained by the gold standard method (broth microdilution). The minimum inhibitory concentration (MIC) found for Candida spp. ranged from 0.125 to $32.0 \mu \mathrm{g} / \mathrm{mL}$, and MIC 50 and MIC 90 were 0.5 and $4.0 \mu \mathrm{g} / \mathrm{mL}$, respectively. For Candida albicans, the MIC range was 0.125 to $1.0 \mu \mathrm{g} / \mathrm{mL}$, and MIC 50 and MIC 90 were $0.5 \mu \mathrm{g} / \mathrm{mL}$ and $1.0 \mu \mathrm{g} / \mathrm{mL}$, respectively. For the Candida parapsilosis complex, the MIC range was 0.25 to $4.0 \mu \mathrm{g} / \mathrm{mL}$, and MIC 50 and MIC 90 were 1.0 and $2.0 \mu \mathrm{g} / \mathrm{mL}$, respectively. Approximately $87 \%$ of Candida spp. samples were sensitive to fluconazole (all C. albicans and $80 \%$ of nonalbicans Candida isolates).

The categorical agreement between the rapid disk diffusion method and the standard disk diffusion method was approximately $95 \%$ and involved 3 minor errors (5\%) (Figure 1). The kappa coefficient $(K=0.77 ; p<0.001)$ showed strong agreement between these two methods. The 
TABLe 1: Candida species identified directly from 91 blood culture samples from 46 patients.

\begin{tabular}{lccc}
\hline Standard identification & \multicolumn{3}{c}{ Number of isolates } \\
Identification matching & Total tested & 14 & 50.00 \\
Candida albicans & 28 & 2 & 66.67 \\
Candida glabrata & 3 & 3 & 60.00 \\
Candida krusei & 5 & 0 & 0 \\
Candida orthopsilosis & 16 & 25 & 96.15 \\
Candida parapsilosis & 26 & 0 & 0 \\
Candida pelliculosa & 3 & 3 & 30.00 \\
Candida tropicalis & 10 & 47 & 51.64 \\
Total & 91 & & \\
\hline
\end{tabular}

TABLE 2: Distribution of Candida spp. and their fluconazole susceptibility profiles according to the gold standard (broth microdilution). The minimum inhibitory concentration (MIC) found for Candida spp. ranged from 0.125 to $32.0 \mu \mathrm{g} / \mathrm{mL}$, and MIC 50 and MIC 90 were 0.5 and $4.0 \mu \mathrm{g} / \mathrm{mL}$, respectively.

\begin{tabular}{lcccc}
\hline Species & Sensitive isolates & Dose-dependent isolates & Resistant isolates & Total isolates \\
\hline Candida albicans & 22 & 0 & 0 & 0 \\
Candida dubliniensis & 1 & 0 & 2 & 22 \\
Candida glabrata & 0 & 0 & 2 & 2 \\
Candida krusei & 0 & 0 & 0 & 2 \\
Candida orthopsilosis & 9 & 2 & 0 & 11 \\
Candida parapsilosis & 15 & 1 & 0 & 1 \\
Candida pelliculosa & 1 & 0 & 0 & 7 \\
Candida tropicalis & 6 & 1 & 4 & 62 \\
Total & 54 & 4 & & 1 \\
\hline
\end{tabular}

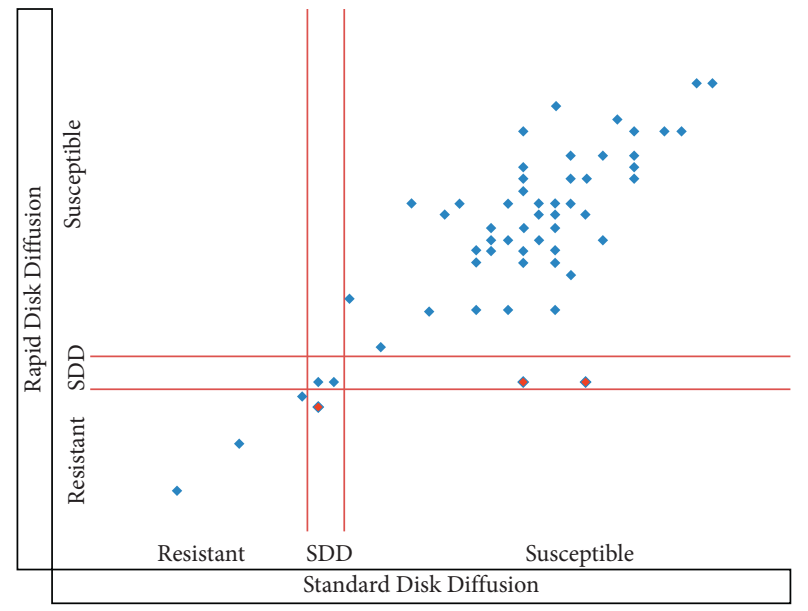

Figure 1: Distribution of susceptible profiles of Candida spp. according to the standard and rapid disk diffusion methods. SDD: fluconazole susceptible-dose dependent.

categorical agreement between the rapid disk diffusion method and the broth microdilution method was $97 \%$ and involved 2 minor errors (3\%) (Figure 2). The kappa coefficient $(K=0.86 ; p<0.001)$ showed almost perfect agreement between these methods. A comparison of the results of the rapid disk diffusion method, the standard disk diffusion method, and the broth microdilution method (gold standard) is shown in Figures 1 and 2.

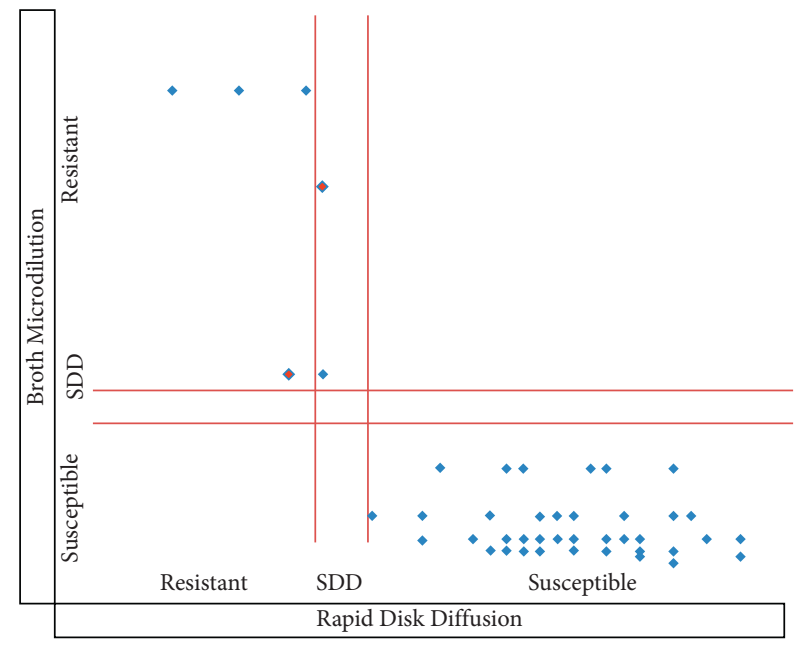

FIGURE 2: Distribution of susceptible profiles of Candida spp. according to the broth microdilution method and the rapid disk diffusion method. SDD: fluconazole susceptible-dose dependent.

\section{Discussion}

The classical diagnostic workflow takes up to several days due to the slow growth of yeasts. The overall performance of our standard identification (Bruker Biotyper and VITEK MS) was in accordance with published data, with $70.7 \%$ of yeast correctly identified to the species, genus, or complex 


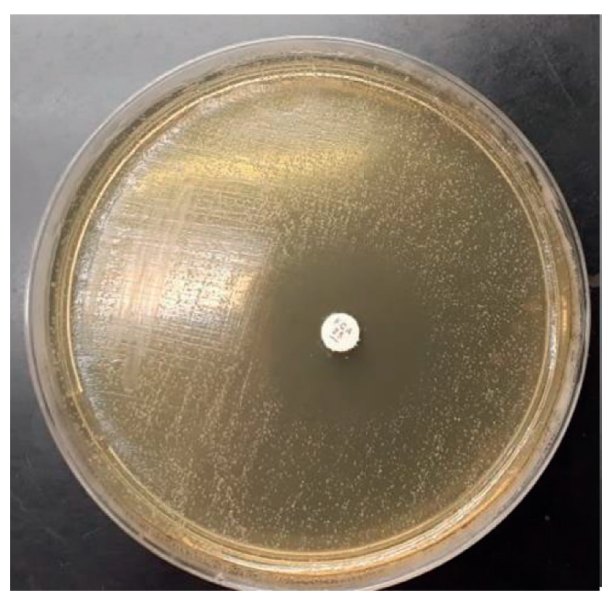

(a)

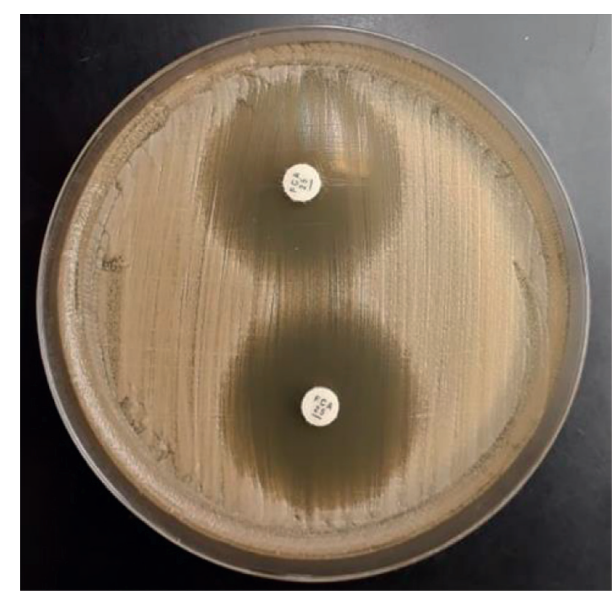

(b)

Figure 3: Comparison between standard disk diffusion (a) and rapid disk diffusion (b) susceptibility testing of Candida spp. for fluconazole.

level [12]. Unlike in the study conducted by Lévesque et al., the overall identification rate for our rapid method was lower than expected (52\%) [13]. These authors used the Bruker Biotyper MALDI-TOF system (Daltonik GmbH, Leipzig, Germany) and obtained identification rates of $95.9 \%$ for C. albicans and $86.5 \%$ for non-albicans Candida species.

The ability to rapidly identify Candida species may be useful to promptly streamline the development of antifungal therapy based on empirical evidence $[14,15]$. However, the emergence and spread of fluconazole-resistant Candida have introduced a pressing need for rapid antifungal susceptibility tests [16]. Our rapid disk diffusion method was reproducible, yielding concordant results and few errors compared to standard disk diffusion and broth microdilution methods. For our method, there were three minor errors compared to the standard disk diffusion method for the C. parapsilosis complex, two minor errors compared to broth microdilution for C. glabrata and C. parapsilosis, no errors for C. albicans isolates (which have the highest incidence in the hospital, $35 \%$ ), and no errors for C. krusei and C. tropicalis. Moreover, in this study, the rapid disk diffusion method was more reliable for broth microdilution, which is the gold standard, than for standard disk diffusion, exhibiting a smaller number of errors, a higher kappa, and a higher categorical agreement rate.

The results indicate that the rapid disk diffusion test is promising for testing additional antifungal agents in microbiology laboratories, given that it can shorten the time needed for the identification of Candida spp. susceptibility profiles by up to two days [17]. This direct method saved on average $21.5 \mathrm{~h}$ for identification and $12.1 \mathrm{~h}$ for susceptibility testing compared to standard methods. The test is practical, easy to use, inexpensive, and rapid. It eliminates process steps, and interpreting halos is clearer and safer. Therefore, with this method, the halo is better delimited, which prevents conflicting results and interoperator error, as shown in Figure 3. By releasing susceptibility profile results more quickly, harm from inappropriate and sometimes ineffective pharmacotherapy can be reduced, aiding in patient recovery and reducing mortality and the length of stay, thus contributing to better patient safety. Further studies of susceptibility testing for other antifungal agents including echinocandins are necessary.

\section{Data Availability}

The data used to support the findings of this study are included within the article.

\section{Conflicts of Interest}

The authors declare no conflicts of interest.

\section{Acknowledgments}

This study was supported in part by $\mathrm{CNPq}$ (Conselho Nacional de Desenvolvimento Científico e Tecnológico, Brazil). The authors would like to thank the Fundo de Incentivo à Pesquisa (FIPE/HCPA).

\section{References}

[1] M. Bassetti, M. Merelli, E. Righi et al., "Epidemiology, species distribution, antifungal susceptibility, and outcome of candidemia across five sites in Italy and Spain," Journal of Clinical Microbiology, vol. 51, no. 12, pp. 4167-4172, 2013.

[2] O. Lortholary, C. Renaudat, C. Renaudat et al., "Worrisome trends in incidence and mortality of candidemia in intensive care units (Paris area, 2002-2010)," Intensive Care Medicine, vol. 40, no. 9, pp. 1303-1312, 2014.

[3] I. H. Peron, F. Reichert-Lima, A. F. Busso-Lopes et al., "Resistance surveillance in Candida albicans: a five-year antifungal susceptibility evaluation in a Brazilian university hospital," PLoS One, vol. 11, no. 7, Article ID e0158126, 2016.

[4] M. Kollef, S. Micek, N. Hampton, J. A. Doherty, and A. Kumar, "Septic shock attributed to Candida infection: importance of empiric therapy and source control," Clinical Infectious Diseases, vol. 54, no. 12, pp. 1739-1746, 2012.

[5] J. A. Cortés, P. Reyes, C. H. Gómez et al., "Clinical and epidemiological characteristics and risk factors for mortality in patients with candidemia in hospitals from Bogotá, 
Colombia," Brazilian Journal of Infectious Diseases, vol. 18, no. 6, pp. 631-637, 2014.

[6] N. Ghanem-Zoubi, D. Zorbavel, J. Khoury et al., "The association between treatment appropriateness according to EUCAST and CLSI breakpoints and mortality among patients with candidemia: a retrospective observational study," European Journal of Clinical Microbiology \& Infectious Diseases, vol. 37, no. 12, pp. 2397-2404, 2018.

[7] M. Puig-Asensio, J. Pemán, R. Zaragoza et al., "Impact of therapeutic strategies on the prognosis of candidemia in the ICU," Critical Care Medicine, vol. 42, no. 6, pp. 1423-1432, 2014.

[8] CLSI, M44-A2: Method for Antifungal Disk Diffusion Susceptibility Testing of Yeasts; Approved Guideline-Second Edition, Wayne, PA, USA, 2009.

[9] European Committee on Antimicrobial Susceptibility Testing, Antifungal Agents-Breakpoint Tables for Interpretation of MICs, European Committee on Antimicrobial Susceptibility Testing, Växjö, Sweden, 2020.

[10] T. Spanu, B. Posteraro, B. Fiori et al., "Direct maldi-tof mass spectrometry assay of blood culture broths for rapid identification of Candida species causing bloodstream infections: an observational study in two large microbiology laboratories," Journal of Clinical Microbiology, vol. 50, no. 1, pp. 176-179, 2012.

[11] K. Jabeen, H. Kumar, J. Farooqi, R. Mehboob, M. E. Brandt, and A. Zafar, "Agreement of direct antifungal susceptibility testing from positive blood culture bottles with the conventional method for Candida species," Journal of Clinical Microbiology, vol. 54, no. 2, pp. 343-348, 2016.

[12] International Organization for Standardization (ISO), Clinical Laboratory Testing and In Vitro Diagnostic Test Systems. Susceptibility Testing of Infectious Agents and Evaluation of Performance of Antimicrobial Susceptibility Test Devices. Part 2: Evaluation of Performance of Antimicrobial, ISO, Geneva, Switzerland, 2007.

[13] S. Lévesque, P. J. Dufresne, H. Soualhine et al., "Lefebvre B, a side by side comparison of bruker biotyper and VITEK MS: utility of MALDI-TOF MS technology for microorganism identification in a public health reference laboratory," PLoS One, vol. 10, Article ID e0144878, 2015.

[14] E. A. Idelevich, B. Grünastel, and K. Becker, "Rapid detection and identification of candidemia by direct blood culturing on solid medium by use of lysis-centrifugation method combined with matrix-assisted laser desorption ionization-time of flight mass spectrometry (MALDI-TOF MS)," Journal of Clinical Microbiology, vol. 55, no. 1, pp. 97-100, 2017.

[15] C. Marinach-Patrice, A. Fekkar, R. Atanasova et al., "Rapid species diagnosis for invasive candidiasis using mass spectrometry," PLoS One, vol. 5, no. 1, Article ID e8862, 2010.

[16] M. A. Pfaller and D. J. Diekema, "Epidemiology of invasive candidiasis: a persistent public health problem," Clinical Microbiology Reviews, vol. 20, no. 1, pp. 133-163, 2007.

[17] Z. A. Khan, M. F. Siddiqui, and S. Park, "Current and emerging methods of antibiotic susceptibility testing," $D i$ agnostics, vol. 9, no. 2, p. E49, 2019. 